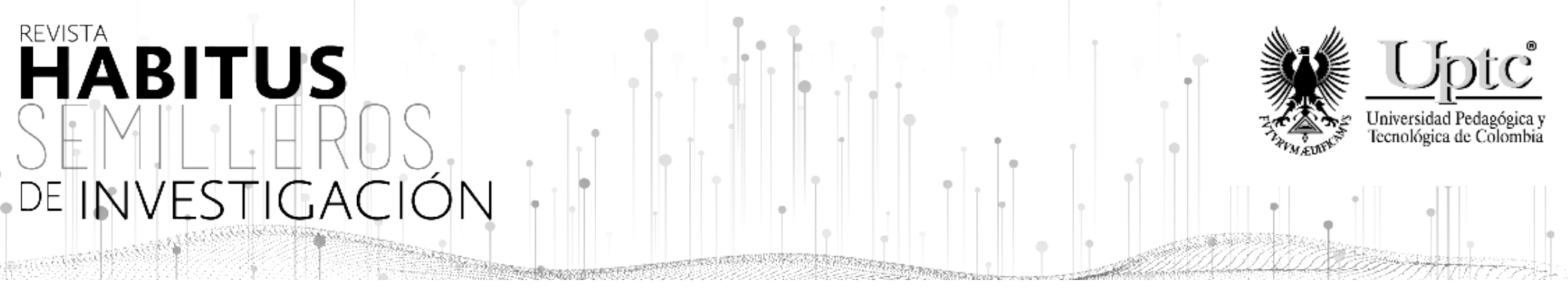

2021 | VOL. 1, NÚM. 1 | ISSN: 2745-2166 | https://doi.org/10.19053/22158391.13316

ARTICULOS ORIGINALES

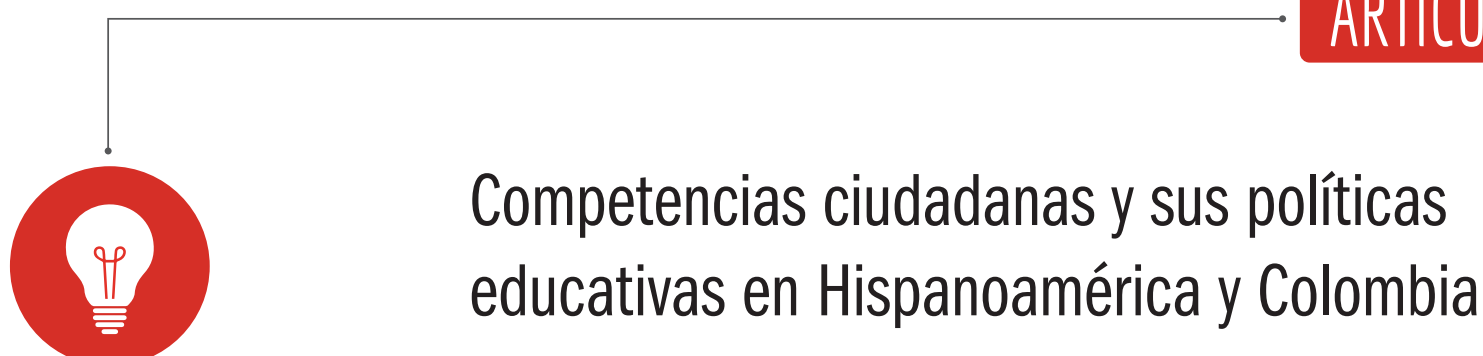

¿. DEISCY SORAYA MONTANAA CONTRERASA

ANGELA LÓPEZ GUIOB

\section{CÓMO CITAR}

Montaña, D., \& López, A. (2021).

Competencias ciudadanas $y$

sus políticas educativas en

Hispanoamérica y Colombia.

Revista Habitus: Semilleros de

investigación, 1(1), e13316. https://doi.

org/10.19053/22158391.13316
RESUMEN: El artículo muestra una revisión documental sobre las competencias ciudadanas y las políticas públicas en Colombia y otros países hispanoamericanos, mediante la recopilación de artículos y el análisis de los discursos de las competencias como saber escolar. Desde una perspectiva metodológica, se rastrearon y se conceptualizaron categorías como: competencias, educación y políticas públicas. Se trabajó en tres etapas: la primera fue un acercamiento histórico y conceptual de las competencias ciudadanas a partir de los años 80 en Colombia y desde la promulgación de la constitución de 1991; en la segunda, se estudió el concepto de ciudadanía desde la vinculación del discurso teórico y experiencias en el aula en diferentes países de habla hispana; y finalmente, se evaluaron las implicaciones que el modelo neoliberal ha tenido tanto en las políticas educativas como en la formación de ciudadanos y su intervención en la educación superior. Como resultado, en el análisis de la categoría de políticas públicas y las competencias ciudadanas, se infiere una importante preocupación por enlazar de manera articulada y permanente el currículo educativo y los lineamientos establecidos por entes gubernamentales a la aplicación y regulación de dichas competencias, no como una cátedra más en las instituciones educativas establecidas para el mercado laboral, sino como un componente formativo fundamental para la construcción de ciudadanía, sin caer en la instrumentalización y en la ambigüedad de la noción de competencias y ciudadanía en el ámbito educativo actual.

PALABRAS CLAVE: políticas públicas, competencias, ciudadanías, escuela, neoliberalismo, Colombia
RECIBIDO: 18/08/2021 - EVALUADO: 14/08/2021

APROBADO: 15/09/2021 - PUBLICADO: 25/10/2021

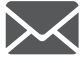

Autor para correspondencia. deiscy.montana@uptc.edu.co
Universidad Pedagógica y Tecnológica de Colombia (Colombia). https://orcid.org/0000-0003-0875-8548

https://orcid.org/0000-0001-9349-4406 


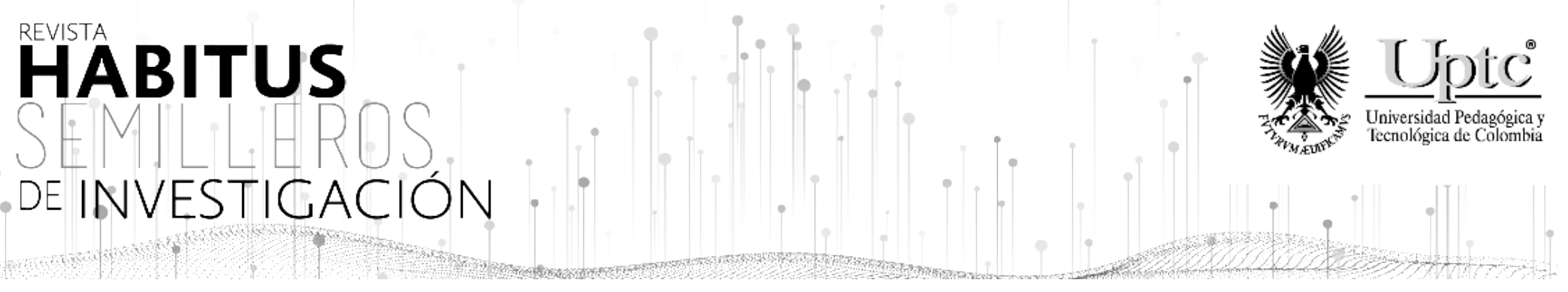

2021 | VOL. 1, NÚM. 1 | ISSN: 2745-2166 | https://doi.org/10.19053/22158391.13316

\section{HOW TO CITE}

Montaña, D., \& López, A. (2021).

Citizenship skills and their educational policies in Latin America and Colombia. Revista Habitus: Semilleros de investigación, 1(1), e13316. https://doi. org/10.19053/22158391.13316

\section{Citizenship skills and their educational policies in Latin America and Colombia}

ABSTRACT: This article shows an overview on citizenship competencies and public policies in Colombia and other Latin American countries, by compiling articles and analyzing the discourses of competencies as common knowledge. From a methodological perspective, categories such as: competencies, education, and public policies were tracked and conceptualized. The study was performed in three stages: the first was a historical and conceptual approach to citizenship competencies from the 80's in Colombia and from the enactment of the Constitution in 1991; the second dealt with the concept of citizenship which was studied from the connection of theoretical discourse and experiences in classroom of different Spanish-speaking countries; and finally, the implications that the neoliberal model has had both in educational policies and in the formation of citizens and their intervention in higher education were evaluated. As a result, in the analysis of the category of public policies and citizenship competencies, there is an important concern to link in an articulated and permanent manner the educational curriculum and the guidelines established by governmental entities to the application and regulation of such competencies, not as just another lecture in the educational institutions established for the labor market, but as a fundamental formative component for the construction of citizenship, without falling into the instrumentalization and ambiguity of the notion of competencies and citizenship in the current educational environment.

KEYWORDS: public policies, competencies, citizenships, school, neoliberalism, Colombia

\section{Competências cidadãs e políticas educacionais na América Latina e na Colombia}

RESUMO: 0 artigo mostra uma revisão documental das competências de cidadania e políticas públicas na Colômbia e em outros países latino-americanos, através da compilação de artigos e da análise dos discursos de competências como conhecimento escolar. De uma perspectiva metodológica, categorias como competências, educação e políticas públicas foram traçadas e conceitualizadas. Trabalhamos em três etapas: a primeira foi uma abordagem histórica e conceitual das competências de cidadania desde os anos 80 na Colombia e desde a promulgação da Constituição de 1991; na segunda, estudamos o conceito de cidadania a partir da ligação entre o discurso teórico e as experiências em sala de aula em diferentes países de língua espanhola; e finalmente, avaliamos as implicações que o modelo neoliberal teve tanto nas políticas educacionais quanto na formação dos cidadãos e sua intervenção no ensino superior. Como resultado, na análise da categoria de políticas públicas e competências de cidadania, inferese uma importante preocupação em vincular de forma articulada e permanente o currículo educacional e as diretrizes estabelecidas pelos órgãos governamentais à aplicação e regulamentação dessas competências, não como mais uma palestra nas instituições educacionais estabelecidas para o mercado de trabalho, mas como um componente formativo fundamental para a construção da cidadania, sem cair na instrumentalização e ambigüidade da noção de competências e cidadania na esfera educacional atual.

PALAVRAS-ChAVE: políticas públicas, competências, cidadania, escola, neoliberalismo, Colombia 
Los resultados esbozados en el presente artículo responden a la revisión documental para el desarrollo del proyecto de investigación titulado Competencias ciudadanas y capital humano: reflexiones sobre la dimensión educativa del neoliberalismo en Boyacá, que pretende mostrar la implicación de las políticas públicas educativas en la formación de los sujetos desde las competencias ciudadanas en el contexto hispanoamericano y, en específico, sus efectos en Colombia. Desde la promulgación de la Constitución Política de 1991, hubo una serie de reformas al sistema educativo para transformar la educación puramente cívica al plano de la enseñanza de las competencias ciudadanas. En el artículo 41 se instituye la enseñanza y el estudio tanto de la Constitución Política como de la formación ciudadana. Se puso de manifiesto que en las instituciones educativas el estudio de esta era obligatorio, como se observa en los trabajos Maestro: condición social y profesión docente en Colombia, 1991-2002 (Castro et al., 2007), Maestro contemporáneo en Colombia: condiciones y configuraciones (Cristancho, 2018) y La "Escuela" en la formación de ciudadanos (Avendaño et al., 2000), que sirvieron de punto de partida para realizar la investigación sobre la condición actual de las prácticas educativas enfocadas en la competencia ciudadana en Colombia.

Las instituciones educativas, en todos los niveles de formación, en particular las de educación superior, vinculan las competencias ciudadanas en su misión y las llaman formación integral. Por ello, se pueden entender las competencias como la suma de tres saberes básicos: el saber, el saber ser y el saber hacer (Rodríguez, 2007). Esto pone de manifiesto la importancia de los parámetros, los lineamientos y las orientaciones que a nivel gubernamental se desarrollan en la educación, y también de su aplicación en las aulas de clase por parte de los docentes. Por lo tanto, es necesario conocer cuáles son los discursos que plantea el Estado colombiano en sus políticas públicas frente a la formación de ciudadanos, específicamente sobre las competencias ciudadanas. Para ello, se elaboró un rastreo de las categorías competencias ciudadanas, educación y política en los discursos analizados, las cuales están presentes en las investigaciones sobre experiencias educativas y políticas de los países hispanoamericanos. Se profundizó en el análisis de relación entre políticas públicas y competencias ciudadanas en Colombia. El análisis de datos se llevó a cabo en revistas como Praxis \& Saber, Revista Educación y Pensamiento, Educación y Educadores, Revista Interamericana de Educación para la Democracia, entre otras. Luego, se revisó el contenido de los textos a través de fichas temáticas y analíticas, de series conceptuales y de la categorización de los conceptos teóricos. Se finalizó con un proceso de interpretación del discurso en torno a los conceptos de competencias 
ciudadanas y políticas públicas, que permitió observar los puntos de conexión de las categorías con el modelo neoliberal y capital humano.

Como consecuencia de lo anterior y para el desarrollo de esta revisión teórica, se identificaron y se clasificaron algunas líneas conceptuales, donde se abordan los estudios de las competencias, las competencias ciudadanas y la formación de sujetos escolares. La estructura del texto está dividida en tres planos de análisis: Política educativa y formación ciudadana; Estrategias, experiencias y competencias ciudadanas; y finalmente, Competencias ciudadanas y educación superior.

\section{Política educativa y formación ciudadana}

En la primera categoría del análisis teórico se hizo un recorrido por diversos textos que trabajan perspectivas históricas, normativas y conceptuales sobre las competencias ciudadanas y la ciudadanía, tanto en los ámbitos nacional como internacional. Navarro-Medina (2015) presenta una amplia conceptualización frente a la noción de ciudadanía, su recorrido y su evolución histórica en España. Afirma que instruir ciudadanos no se trata solo de educarlos, sino de formarlos activos y participativos en la esfera pública. De la misma forma, Villera y Lobo (2017) presentan referentes conceptuales sobre lo que significan la ciudadanía, las competencias ciudadanas y sus implicaciones para la paz.

Al abordar las competencias ciudadanas, es necesario acercarse a los procesos de evolución, a los cambios históricos que han sufrido tanto el concepto como las instituciones y a la forma en que la normatividad ha asumido dicha transformación.

Para el caso colombiano, el modelo educativo por competencias se acogió desde finales del siglo XX y se materializó a través de las diversas políticas educativas establecidas hasta hoy (Álvarez \& Henao, 2019). Así, la normativa dictada en el sistema educativo es fundamental para el ámbito jurídico, para el desarrollo de los diversos programas pedagógicos y para la convivencia desde un enfoque de derechos, organizados por el Ministerio de Educación Nacional (MEN). Sin embargo, no brinda las herramientas

suficientes a las instituciones y a los docentes para intervenir eficazmente en las relaciones pacíficas en la comunidad educativa (Salas \& Cómbita, 2017).

En Colombia, a partir del año 2000, el sistema educativo tuvo una reorganización desde las reformas educativas de acuerdo con el nuevo modelo implementado y desplazó sus categorías hacia unos objetivos concretos de alineación con las políticas de gobierno para introducir cambios según el orden social y las tensiones presentes entre la economía, el Estado 
y la sociedad, como señalan Álvarez y Henao (2019), quienes resaltan las repercusiones de la reforma educativa en la Ley General de Educación, la cual estableció el modelo de institución educativa y dio prioridad a lo mercantil por encima de lo formativo en la organización escolar desde la oferta y la demanda. Así, esta la Ley sufrió una afectación en su autonomía y sus lineamientos (Álvarez \& Henao, 2019) al transformarse en un proceso educativo orientado por criterios financieros. De esta manera, a partir de 2002 se configuró un modelo educativo por competencias en Colombia, con el propósito de obtener fines inmediatos en un modelo con bases neoliberales, centrado en el mercado y en las demandas del capital humano, acompañado del ideal de crecimiento económico. En consecuencia, se "afectan de manera negativa el desarrollo humano y el bienestar integral de la sociedad" (Álvarez \& Henao, 2019, p. 28).

Educar a los estudiantes en su papel como ciudadanos es una enorme tarea que no puede ser vinculada, exclusivamente, a una materia en particular, sino a todo el sistema educativo en general, pues no ha conseguido formar a los jóvenes en los conocimientos, actitudes y competencias que les permitan participar activamente de la sociedad. Nos encontramos quizás ante un modelo de escuela más preocupado por las calificaciones que obtienen los estudiantes, por acreditar unos conocimientos, que presenta dificultades para que los jóvenes desarrollen su educación ciudadana, para que se eduquen a través de la enseñanza escolar en aquellas capacidades que les permitan fundamentar su participación social. (Navarro-Medina, 2015, p. 58)

De igual manera, en los escritos de Vega y Castro (2006), de Mieles y Alvarado (2012) y de Niño (2017), se reconoce especialmente el concepto de ciudadanía desde la multiculturalidad y sus implicaciones tanto en la mirada urbano-rural como en las diversas etnias indígenas y afrocolombianas que habitan en el país, con lo que se profundiza en el tipo de ciudadano que desde la diversidad se está formando en el contexto local.

En el rastreo bibliográfico se encontraron varios textos que analizan la formación de competencias ciudadanas desde la promulgación de la Constitución de 1991, entre ellos destacan:

- Formación en competencias ciudadanas y cultura política (Álvarez \& Henao, 2019).

- Estándares básicos en competencias ciudadanas: una aproximación al problema de la formación ciudadana en Colombia (Restrepo, 2006).

- La formación ciudadana en la educación obligatoria en Colombia: entre la tradición y la transformación (González-Valencia \& Santisteban-Fernández, 2016). 
- Desarrollo histórico, teórico y conceptual de las competencias ciudadanas y las capacidades ciudadanas en el modelo educativo distrital de Bogotá (Castañeda, 2016).

Estos realizan un acercamiento teórico y conceptual a la ciudadanía desde la normativa nacional, pero aplicada específicamente al caso de la capital. Así mismo, Rincón (2017) retoma esta perspectiva de estudio, pero desde un enfoque de la pedagogía católica. Sobre el campo normativo más específicamente, se encuentran los escritos:

- Análisis de la convivencia escolar desde la perspectiva psicológica, legal y pedagógica en Colombia (Salas \& Cómbita, 2017).

- Miradas sobre los lineamientos curriculares y los estándares básicos de competencias en Colombia. Estado del arte 2002-2016 (D. Hernández, 2017).

- Competencias ciudadanas y construcción de ciudadanía juvenil (Murillo \& Castañeda, 2007).

En ellos se analiza el surgimiento de las competencias ciudadanas al tener en cuenta los Estándares Básicos de Competencias y los Lineamientos Curriculares erigidos por el MEN (2004).

Desde la instancia de las políticas públicas a nivel educativo empieza a apreciarse a partir de este momento una desarticulación entre escuela y familia, en donde, por ley, ambas tienen como deber la formación integral de los niños/as y adolescentes, en la práctica no existe una normatividad clara dirigida a la familia para que cumpla con dicha función. A nivel educativo, la vigilancia del Estado recae fundamentalmente para la escuela, pero ¿quién vigila a la familia en el cumplimiento de su rol en la educación? (Salas \& Cómbita, 2017, p. 84)

Así, para Salas y Cómbita (2017), la formación en ciudadanía es uno de los pilares fundamentales en el desarrollo del currículo dirigido por el MEN en las ciencias básicas, ya que los Estándares Básicos en Competencias Ciudadanas establecen los contenidos temáticos a trabajar, así como la clasificación y los lineamientos para desarrollarlas según el grado escolar que se encuentra el estudiante.

Por otra parte, Avendaño et al. (2015) hacen una revisión documental de los textos propuestos por el MEN sobre competencias ciudadanas, para posteriormente formular un análisis crítico de este programa a partir de la identificación del saber como un instrumento mercantil. Así mismo, Torres y Reyes (2015) analizan el programa de competencias ciudadanas como una técnica de gobierno y de disciplina que potencia la formación de los estudiantes como capital humano y genera un emplazamiento de las 
dinámicas neoliberales vinculadas a las normas y políticas educativas que se ejercen en la escuela y en la formación del sujeto. Así "la fragmentación del proceso pedagógico conlleva... que se instrumentalice la competencia porque con frecuencia se enseña, se aprende y se evalúa de diferente manera" (Álvarez \& Henao, 2019, p. 29).

En el ámbito histórico, Muñoz (2013) analiza la idea de ciudadano en el periodo comprendido entre 1910 y 1948 en Colombia. Busca apreciar cómo el Estado, la Iglesia, los partidos políticos, las familias y la comunidad en general recibieron y adoptaron la iniciativa de implantar los manuales de historia, de instrucción cívica y de urbanidad. La autora realizó la búsqueda de cuarenta manuales, que fueron clasificados y referenciados. También, en España se desarrolló un proyecto histórico similar, donde se analizaron manuales escolares de historia y su incidencia en la formación de los estudiantes, proyecto que fue rescatado por Tiana $(2000,2005)$, quien hace referencia a la importancia que de tiempo atrás tiene la enseñanza de la cívica dentro de los contextos escolares.

En general, estas investigaciones presentan un panorama histórico en los ámbitos nacional e internacional, mediante la implementación de nuevas políticas educativas y normativas que buscan responder a las necesidades del mercado en los contextos escolares de finales del siglo XX, como la obligatoriedad del desarrollo de competencias y específicamente de las competencias ciudadanas en las instituciones educativas. Se analiza la desconexión entre el MEN y las instituciones educativas por la falta de orientación en la aplicación de nuevas cátedras, tendientes a un real y efectivo cumplimiento del objetivo de la política pública. Asimismo, a nivel internacional, se observan un avance en la conceptualización de ciudadanía como factor determinante en la educación y el compromiso por parte de docentes y directivos que exige el desarrollo de unos estándares que permitan una participación activa de los sujetos escolares en la esfera pública y social.

\section{Estrategias, experiencias y competencias ciudadanas}

Uno de los resultados esperados con la aplicación de las competencias ciudadanas es disminuir el impacto de las problemáticas sociales, como la violencia, e incrementar la participación democrática desde grados escolares iniciales hasta la participación en espacios más amplios en los últimos grados de la educación media. Por eso, Prada (2020) señala que esta responsabilidad no solo involucra a la escuela, sino también a su contexto como un espacio de interacción social. Además, en su trabajo cualitativo analiza el vínculo, la pertinencia y la concordancia del diseño curricular del Ministerio Nacional de 
Educación de la República Dominicana con las clases de educación física en ciclo secundario. En el informe, resalta la disonancia entre los objetivos curriculares de la asignatura enfocada a las competencias ciudadanas y los objetivos que el grupo docente imparte en el aula. Resalta una desconexión que puede estar presente no solo en República Dominicana, sino también en otros países de América, incluida Colombia.

Desde otra perspectiva, a inicios del siglo XX la educación tuvo que pensar un factor clave para el futuro y para la subsistencia de la humanidad: el medioambiente. La relación que tenemos con el entorno se volvió un tema central e importante para la formación de sujetos conscientes de que el daño al medioambiente tiene un efecto global irreversible. En este contexto, es importante que el modelo educativo potencie las capacidades de los estudiantes, particularmente en el desarrollo de sus competencias, para lograr un vínculo entre lo individual y lo social, como lo mencionan García y González (2014). Por ello, el modelo educativo tendrá que enfocarse en los retos y problemáticas que enfrentan los ciudadanos del siglo XXI, lo que demanda una integración de las TIC y de las estrategias de protección ambiental. Las competencias en las instituciones se vuelven una capacidad adaptativa para responder a necesidades específicas que enfrentan en su contexto sociocultural. Según Santander et al. (2018), las competencias ciudadanas son un componente que promueve hábitos favorables hacia el medioambiente. Por esta razón, se invita al fortalecimiento de aptitudes que permitan crear conciencia sobre el cuidado del medioambiente, convertirlo en un nuevo elemento para su resignificación e invitar a personas de otros perfiles profesionales al trabajo en pro de estas iniciativas.

En educación, una estrategia es la habilidad de dirigir planes formativos para conseguir unas metas de aprendizaje. Por eso, en las competencias ciudadanas estos logros deben girar en torno a la promoción de la convivencia pacífica y a la formación del estudiante. La segunda categoría de análisis que se identificó en esta temática es la aplicación de estrategias que buscan el afianzamiento de las competencias ciudadanas a través de varias experiencias, entre las que se encuentran:

○ el fortalecimiento de espacios de participación - por ejemplo, el uso de plataformas virtuales como las redes sociales- (Rojas et al., 2018).

- la evaluación de estrategias en aplicación del aprendizaje cooperativo (Salmerón, 2010).

- el establecimiento de grupos de cultura para mejorar las prácticas de grupos sociales que conviven en la Amazonía colombiana (Cruz, 2008). 
- la promoción y la convivencia pacífica a partir de estrategias dirigidas a estudiantes tanto en el aula como en los hogares mediante la constante comunicación con las familias (Ramos et al., 2007).

- las modificaciones en los currículos escolares (Baracaldo, 2014; Otálora, 2018).

- la utilización de los pactos de aula como herramienta didáctica en la mediación y solución de conflictos entre estudiantes (Rodríguez, 2013).

- el manejo de las TIC para establecer competencias sociales (Contreras, 2009).

Otra de las iniciativas halladas es la liderada por Díaz (2017), la cual se centra en la aplicación de las competencias ciudadanas para resignificar los ambientes escolares en la ciudad de Ibagué, Colombia. Tal investigación se centró en analizar las principales problemáticas que aquejan a la comunidad escolar en una institución específica, para obtener cambios en la mentalidad de los docentes y sus modificaciones en las prácticas educativas con los estudiantes. Otro ejemplo se observa en España, donde se desarrolló una propuesta novedosa con el uso de libros de texto como aporte importante en la formación de ciudadanos, la cual fue estudiada por Miralles (2009). Además, se encontró un artículo que permite observar la importancia del aporte de las familias en la educación de los estudiantes a través de los estilos educativos paternos (Cerezo et al., 2011).

Quiroz y Jaramillo (2009) presentan una reflexión teórica y llaman la atención frente a la necesidad de la educación cívica para la formación de ciudadanía desde la totalidad de involucrados en el sector educativo, sin importar el área del saber en que se desempeñen. Por su parte, los aportes presentados por Geijsel et al. (2012), Londoño y Rojas-Devia (2020) TorneyPurta et al. (1999) y D. Hernández (2017) muestran experiencias aplicadas con jóvenes de diversos países, donde se cuestionan sobre los conocimientos y las perspectivas de principios democráticos, la confianza en el gobierno y los niveles de compromiso de las instituciones educativas en la formación de ciudadanía y de democracia.

En la ciudad de Santa Marta, Barrera et al. (2011) buscaron analizar la formación ciudadana desde el contexto social específico de estudiantes de grado undécimo. Por este motivo, es necesario emprender la formación en competencias desde la educación inicial, así como menciona Rojas (2018), quien resalta que la formación en competencias ciudadanas es una herramienta de aprendizaje que debe estar basada en valorar la cultura, las raíces, la historia y la institucionalidad. Asimismo, Mejía y Perafán (2006) presentan una propuesta crítica frente a las competencias, donde no se 
suscita el cambio de mentalidad de los estudiantes y docentes, sino la adopción de una postura de cuestionamiento hacia ella, para desarrollar el pensamiento reflexivo y autónomo referente al tema de la ciudadanía.

El informe Education for citizenship and the teaching of democracy in schoo/ (Membership of the Advisory Group, 1998) presenta una iniciativa del Reino Unido que sugiere la necesidad de trazar lineamientos mediante una política nacional, con el fin de empoderar a los jóvenes en la participación como agentes activos, informados, críticos y responsables. Por otro lado, la iniciativa de aulas flexibles de Montoya et al. (2018) exhibe un espacio de reflexión frente al fortalecimiento de las competencias ciudadanas desde la resignificación del lenguaje oral.

A partir de estas investigaciones, se puede inferir la desarticulación entre los objetivos curriculares de las diversas instituciones educativas y los lineamientos de las competencias ciudadanas frente a los resultados observados en las sociedades de los diferentes países de Hispanoamérica e incluso de habla inglesa. Se analiza que las diversas estrategias y experiencias adoptadas para el desarrollo de las competencias ciudadanas son ineficientes, ya que las condiciones de vida de los educandos son un factor fundamental para la formación de reales ciudadanos competentes, que a la postre muestren preocupación por mejorar tanto en el plano ambiental como en el social. Se debe pensar más allá de la creación de proyectos de participación ciudadana, cátedra para la paz y libros de texto sobre formación ciudadana, cuyos efectos no solo se reflejen en la escuela, sino también en el contexto local y exija la cooperación de las familias en la educación de los jóvenes, para generar habilidades y espacios de participación democrática a la ciudadanía.

Finalmente, se puede comprender que en Colombia las competencias ciudadanas son los conocimientos y las habilidades cognitivas, emocionales y comunicativas que hacen posible que las personas participen en la construcción de una sociedad democrática, pacífica e incluyente. Por ejemplo, el conocimiento sobre los mecanismos constitucionales para proteger los derechos fundamentales, como la tutela y el derecho de petición, es esencial para participar democráticamente y para validar y hacer respetar los derechos humanos.

Al analizar las experiencias de las competencias ciudadanas, resulta importante profundizar en las capacidades para formular distintas alternativas de solución, las cuales son una competencia procedimental que llevaría a la resolución pacífica de conflictos entre personas o grupos. El reconocimiento y el manejo de las emociones propias son una competencia emocional fundamental para relacionarse de manera responsable y tranquila 
con los demás. De la misma forma, se requiere ahondar en la capacidad para escuchar seriamente los puntos de vista divergentes. Este es un ejemplo de competencia comunicativa fundamental para convivir en una sociedad donde se construye la paz a partir de las diferencias (Chaux, 2012).

\section{Competencias ciudadanas y educación superior}

En el tercer plano de análisis se recogen algunas iniciativas que han llamado la atención frente a la necesidad de la continuación en formación de las competencias ciudadanas en el ámbito universitario, como es el caso de Competencias ciudadanas aplicadas a la educación en Colombia mencionada por Rodríguez et al. (2007) sobre el proyecto Tuning', el cual busca que los programas educativos se centren en el desarrollo de las competencias para el aprendizaje y en la apertura de la oferta laboral como estrategia para la construcción de una ciudadanía responsable al extender estos espacios de las competencias a la educación superior. A partir de esto, desde la cualificación profesional inicial, así como desde la formación superior de los estudiantes, se han establecido algunas estrategias para resignificar y potencializar las competencias ciudadanas en las dimensiones personal, interpersonal, social y profesional, como las presentadas por Carrillo (2013).

En respuesta a estas iniciativas, se han desarrollado metodologías que han sido puestas en marcha, como el caso registrado por Ruiz y Chaux en La formación de competencias Ciudadanas (2005), dirigido principalmente a los encargados de la capacitación de maestros, tanto en las facultades de educación como en las escuelas normales de Colombia. Así mismo, en Ciudadanía, diversidad y participación. Educar para la participación desde la diversidad (Hernández, 2012) se generan algunas propuestas para ejecutar esta estrategia en las universidades. También, García y González (2014) pretenden mostrar la ciudadanía como un ejercicio político propiamente dicho, al comprender la profundidad con la que el concepto puede llegar a definirse. Quintero y Molano (2009) realizan una investigación con enfoque crítico que busca describir las concepciones y las creencias que se tienen de la formación en competencias desde la perspectiva universitaria.

Por otra parte, en Latinoamérica, Calderíus y Martínez (2011) buscaron visibilizar el progreso de las competencias ciudadanas en la educación

1 Creado en el año 2000, a partir de la Declaración de Bolonia (1999), para pensar la enseñanza superior de forma competente y adaptiva dentro de un grupo de cien universidades para restructurar la educación en Europa, a partir de la promoción de competencias en la formación superior. 
superior cubana, al aportar un método y procedimientos que contribuyen al proceso formativo de los profesionales, basado en el perfeccionamiento de la democracia socialista e imperante en ese país.

Ahora bien, en Colombia ha habido varios estudios con algunas estrategias frente al fortalecimiento de las competencias sociales, gracias a la diversidad étnica y cultural que se congrega en las universidades. Por ejemplo, Cabrera y Gómez (2019) buscaron potencializar en el ciudadano capacidades que le permitan consolidarse como agente responsable de su propio cambio social y de su entorno. Así mismo, se encuentra el estudio de Peñalva y LópezGoñi (2014) en la Universidad Pública de Navarra, España, donde señalan que la aplicación de las competencias ciudadanas en la educación superior implicaría a su vez una capacidad para adaptarse al encuentro de diversas culturas en el que el espacio de socialización genere principios de equidad e igualdad entre migrantes y residentes.

En el trabajo de Rendón (2012) se comprende el fenómeno de las convivencias como construcciones permanentes de las interacciones sociales en los ámbitos regional y nacional. No obstante, en el espacio universitario se privilegia el componente formal y normativo que no permite crear espacios de convivencia. Como resultado se obtiene la falta de reconocimiento entre los sujetos, lo que tiende a generar una gama de emociones, sensaciones y conflictos que estallan con el tiempo.

Por otro lado, a partir del modelo económico actual, se visualiza una barrera al momento de insertar las competencias ciudadanas en la educación superior, debido a que los actores escolares están preocupados por una vinculación a las demandas del mercado laboral y dejan de lado el desarrollo de las competencias ciudadanas, lo cual presenta la relación entre los sujetos y su entorno en una nueva etapa educativa. Según Pulido y Acuña (2014),

E\}es importante comprender cómo se interpreta la inserción del modelo norteamericano en la educación superior ... la adopción de políticas neoliberales inmersas en los procesos de globalización, con las que se ha pretendido modernizar el sistema educativo, al transformar la concepción de educación como un bien público en una especie de mercancía colocada en el escenario del mercado. Estas políticas han planteado una educación cuantificada, en términos de producción, rentabilidad en el mercado, todo esto derivado del modelo económico, que transforma la función social de la universidad. (pp. 235-236)

Así mismo, Agudelo y Chaparro (2019) mencionan que el surgimiento de la universidad en Colombia se organizó de manera articulada con las profesiones, con la transmisión de conocimientos y con la capacitación. 
Sin embargo, con el auge del neoliberalismo en las últimas décadas, la formación se ha reducido, en ocasiones, a la oferta de programas y cursos, a otorgar diplomas y a dar cumplimiento a requerimientos de la política en el campo. Para Foucault (2007), estas prácticas se enmarcan dentro de las características del neoliberalismo norteamericano, en el que se encuentra a su vez la teoría del capital humano, donde el liberalismo es una técnica que se convierte en la forma de ser y de pensar del sujeto escolar contemporáneo. De este modo, la educación sufre un proceso de mutación de sentido y de fines, lo cual se hace manifiesto en su rol subordinado al sistema productivo, por su especialización como institución que dota de competencias a los individuos (Niño et al., 2016) y que reproduce la adaptación de los sujetos como individuos flexibles, al introducir en estos las nuevas reglas del neoliberalismo.

En suma, es notoria una constante preocupación por incentivar las competencias ciudadanas en la educación superior, ya que la transformación de la educación en las últimas décadas inserta a los sujetos en una formación que propende hacia unas competencias básicamente mercantiles que no apuntan a un verdadero desarrollo humano con determinados ideales y valores; en cambio, se preservan al margen de sus beneficios en las estructuras de oferta y demanda los diversos programas educativos que se presentan a través del control institucional.

\section{Conclusiones}

A través de esta revisión documental, se concluyen algunos aspectos por resaltar, que pueden desarrollarse en próximas investigaciones. Se destacan: el impacto de la reforma educativa en el siglo XX y las políticas educativas para Colombia - sus consecuencias en los años posteriores, tanto en la educación básica como superior-; y la orientación de unas competencias ciudadanas eficientes al tomar en consideración proyectos sociales y ambientales que contribuyan a una formación integral del sujeto escolar y a la continuidad del proceso de formación ciudadana en profesionales al encuentro de diversos acontecimientos sociales, culturas y espacios de socialización. Por ello, se pretende que la educación en competencias no se limite a la demanda y a la oferta que exige el mercado laboral, puesto que este busca consolidar una formación desde el capital humano.

En Colombia, desde la Constitución Política de 1991, se evidencia un cambio en la estructura del sistema educativo, en especial desde el aprendizaje del saber al aprendizaje por competencias, entre las que están las competencias ciudadanas de enseñanza obligatoria, donde se encuentran 
algunas contradicciones entre los lineamientos trazados por el MEN y su falta de orientaciones y vinculación en las instrucciones educativas en materia de ciudadanía.

A partir de las tematizaciones logradas y de las fichas temáticas trabajadas, se puede inferir en el análisis de la categoría de políticas públicas una importante preocupación por enlazar de manera articulada y permanente el currículo educativo y los lineamientos establecidos por entes gubernamentales con la aplicación y la regulación de las competencias ciudadanas, no como una cátedra más en las instituciones educativas, sino como un componente formativo fundamental para la construcción de ciudadanía, sin caer en la instrumentalización ni en la ambigüedad de la noción de competencias y ciudadanía en el ámbito educativo actual.

En general, se observa que, en las últimas décadas, la enseñanza de las competencias ciudadanas en diversos países hispanoamericanos ha tenido una aceptación significativa, al plantear que el papel del ciudadano activo se convierte en un proceso clave en el progreso social de un país. Por eso, se incentiva su aplicación de forma transversal a todas las asignaturas impartidas. Sin embargo, su articulación con la escuela y con la familia es cada vez más frágil. Esta separación aparece marcada por el auge de las implicaciones de las políticas económicas en el ámbito educativo, las cuales han formado al sujeto con competencias afines más al mercado laboral que al proceso de formación ciudadana, lo cual crea un capital humano útil al mercado, pero desconectado de su contexto social.

Por lo tanto, es necesario incentivar unas políticas educativas que respondan a la necesidad y a las experiencias del ciudadano desde su cultura y sus necesidades, con el fin de hacer avances importantes en el reconocimiento de las diferentes perspectivas y costumbres que convergen en cada nación. Por esta razón, es necesario extender dichas competencias no solo a la educación básica y media, sino también a la superior, para que el profesional que se configure en estos espacios sea un sujeto con competencias ciudadanas integrales y busque lo mejor para su entorno y para su sociedad.

\section{Referencias}

Agudelo, N., \& Chaparro, H. (2019). La formación de sujetos sociales: contextos escolares pertinentes. Revista Latinoamericana de Estudios Educativos, 15(2), 85-110. https://doi.org/10.17151/ rlee.2019.15.2.5

Álvarez, M., \& Henao, F. (2019). Formación en competencias ciudadanas y cultura política. Revista Educación y Pensamiento, 26(26), 25-38.

Avendaño, A., Cortés, O., \& Castillo, C. (2000). La "Escuela” en la formación de ciudadanos. Nómadas, (13), 223-228. http://nomadas.ucentral.edu.co/index.php/8-articulos/20-tabla-de-contenido-no-13 
Avendaño, E., Gutiérrez, D., \& Malagón, M. (2015). Otra ciudadanía es posible. Análisis crítico al Programa de Competencias Ciudadanas propuesto por el Ministerio de Educación Nacional [Tesis de maestría, Universidad Pedagógica Nacional]. Repositorio Institucional UPN. http://hdl.handle. net/20.500.12209/820

Baracaldo, M. (2014). Fortalecimiento de competencias ciudadanas de convivencia y paz para la administración de conflictos que se presentan entre los estudiantes de décimo de la IED La Gaitana en el ciclo 5 de la jornada mañana. [Tesis de maestría, Universidad Libre]. Repositorio Institucional Unilibre. https://hdl.handle.net/10901/8633

Barrera, D., Madariaga, C., \& Palacio, J. (2011). Análisis de contextos sociales y su incidencia en el desarrollo de competencias ciudadanas en estudiantes de undécimo grado de Santa Marta. Psicogente, 14(26), 235-254. http://revistas.unisimon.edu.co/index.php/psicogente/article/view/1830

Cabrera, M., \& Gómez , F. (2019, 11-12 de julio). Competencias ciudadanas y educación superior, una alternativa al desarro/lo [Ponencia]. V Congreso de Innovación Educativa y Docencia en Red, Universitat Politècnica de València, España. https://doi.org/10.4995/INRED2019.2019.10390

Calderíus, M., \& Martínez, N. (2011). Formación de competencias ciudadanas en las universidades cubanas: una contribución al perfeccionamiento de nuestra democracia. Ciencia en su PC, (3), 109-120.

Carrillo, O. (2013). Comprendiendo la adquisición de las competencias ciudadanas en alumnos de los programas de cualificación profesional inicial. Educar, 49(2), 207-226. https://doi.org/10.5565/rev/ educar.417

Castañeda, J. (2016). El desarrollo histórico, teórico y conceptual de las competencias ciudadanas y las capacidades ciudadanas en el modelo educativo distrital de Bogotá. [Tesis de maestría, Universidad Santo Tomás]. Repositorio Institucional Universidad Santo Tomás. http://dx.doi.org/10.15332/ tg.mae.2016.00482

Castro, J., Pulido, Ó., Peñuela, D., \& Rodríguez, V. (2007). Maestro: condición social y profesión docente en Colombia 1991-2002. UPN; IDEP. https://repositorio.idep.edu.co/bitstream/handle/001/248/ Mestro\%3acondici\%c3\%b3n\%20social\%20y\%20profesi\%c3\%b3n\%20docente\%20en\%20Colombia\%20 1991-2002.pdf?sequence=1\&isAllowed=y

Cerezo, M., Casanova, P., Torre de la, M., \& Carpio, M. (2011). Estilos educativos paternos y estrategias de aprendizaje en alumnos de Educación Secundaria. European Journal of Education and Psychology, $4(1), 51-61$.

Contreras, C. (2009). Alfabetización digital y formación de competencias ciudadanas [Tesis doctoral, Universidad de Barcelona]. Dipòsit Digital de la Universitat de Barcelona. http://hdl.handle. net/2445/43089

Chaux, E. (2012). Educación, Convivencia y Agresión Escolar. Ediciones Uniandes; Taurus; Santillana.

Díaz, A. (2017). Las competencias ciudadanas, como estrategia pedagógica para resignificar los ambientes escolares [Trabajo de grado de especialización inédito]. Universidad del Tolima. https://45.71.7.21/ handle/001/1977

Cristancho, C. (2018). Maestro contemporáneo en Colombia: condiciones y configuraciones. [Tesis de maestría no publicada]. Universidad Pedagógica de Colombia.

Cruz, F. (2008). Educar para gestionar conflictos en una sociedad fragmentada. Una propuesta educativa para una cultura de paz [Tesis doctoral inédita, Universidad de Barcelona]. Repositorio Institucional Universitat de Barcelona. http://diposit.ub.edu/dspace/handle/2445/43079

Declaración de Bolonia. (1999). http://diposit.ub.edu/dspace/handle/2445/43079 http://eees.umh.es/ contenidos/Documentos/DeclaracionBolonia.pdf

Foucault, M. (2007). Nacimiento de la biopolítica: curso en el Collége de France: 1978-1979. (Trad. H. Pons). Fondo de Cultura Económica.

García, G., \& González, C. (2014). Competencias ciudadanas: consideraciones desde el concepto de ciudadanía. P/umilla Educativa, 13(1), 373-396. https://doi.org/10.30554/plumillaedu.13.416.2014

Geijsel, F., Ledoux, G., Reumerman, R., \& Ten, G. (2012). Citizenship in young people's daily lives: Differences in citizenship competences of adolescents in the Netherlands. Journal of Youth Studies, 15(6), 1-19. http://dx.doi.org/10.1080/13676261.2012.671932 
González-Valencia, G., \& Santiesteban-Fernández, A. (2016). La formación ciudadana en la educación obligatoria en Colombia: entre la tradición y la transformación. Educación y Educadores, 19(1), 89 -102. https://educacionyeducadores.unisabana.edu.co/index.php/eye/article/view/5533

Hernández, C. (2012). Ciudadanía, diversidad y participación. Educar para la participación desde la diversidad. Revista Educação e Políticas em Debate, 1(1), 146-157. http://www.seer.ufu.br/index.php/ revistaeducaopoliticas/article/view/17376

Hernández, D. (2017). Miradas sobre los lineamientos curriculares y los estándares básicos de competencias en Colombia. Estado del arte 2002-2016 [Trabajo de grado de especialización inédita, Universidad Pedagógica Nacional]. Repositorio Institucional UPN. http://repository.pedagogica.edu.co/ handle/20.500.12209/9467

Londoño, C., \& Rojas-Devia, J. (2020). Crisis y práctica filosófica en la educación. Praxis \& Saber, 11(25), 153 176. https://doi.org/10.19053/22160159.v11.n25.2020.9910

Mejía, A., \& Perafán, B. (2006). Para acercarse al dragón, para amansarlo, es necesario haberlo amansado primero: una mirada crítica a las competencias ciudadanas. Revista de Estudios Sociales, (23), 23-35.

Membership of the Advisory Group. (1998). Education for citizenship and the teaching of democracy in schools. QCA Publications.

Mieles, M., \& Alvarado, S. (2012). Ciudadanías y competencias ciudadanas. Estudios Políticos, (40), 53-75. https://revistas.udea.edu.co/index.php/estudiospoliticos/article/view/13203

Ministerio de Educación Nacional de Colombia. (2004). Estándares Básicos de Competencias Ciudadanas. https://www.mineducacion.gov.co/1621/article-116042.html

Miralles, P. (2009). La didáctica de la historia en España: retos para una educación de la ciudadanía. En R. Ávila, B. Borghi, \& I. Mattozzi (Eds). La educación de la ciudadanía y la formación del profesorado. Un proyecto educativo para la "Estrategia de Lisboa", (1 a ed. pp. 259-270). Pàtron.

Montoya, A., González, C., \& Osorio, Z. (2018). Aulas flexibles: un espacio de reflexión frente a la resignificación del lenguaje oral en los estudiantes de la I. E. Rafael J. Mejía para el empoderamiento de las competencias ciudadanas [Tesis de maestría inédita, Universidad de Antioquia]. Repositorio Institucional Universidad de Antioquia. https://bibliotecadigital.udea.edu.co/handle/10495/12199

Muñoz, M. (2013). El ciudadano en los manuales de historia, instrucción cívica y urbanidad, 1910-1948. Historia y Sociedad, (24), 215-240. https://revistas.unal.edu.co/index.php/hisysoc/article/view/39775

Murillo, G., \& Castañeda, N. (2007). Competencias ciudadanas y construcción de ciudadanía juvenil. Revista del CLAD, Reforma y Democracia, (37), 1-17.

Navarro-Medina, E. (2015). El desarrollo de las competencias ciudadanas a través de la enseñanza de la historia de España. Enseñanza de las Ciencias Sociales: Revista de Investigación, 14, 49-59.

Niño, J. (2017). Política etnoeducativa y enfoque de competencias: la paradoja del reconocimiento en el marco liberal y capitalista [Tesis de maestría inédita]. Repositorio Institucional Pontificia Universidad Javeriana. https://repository.javeriana.edu.co/handle/10554/21872

Niño, L., Tamayo, A., Gamma, A., \& Díaz, J. (2016). Competencias y currículo. Problemas y tensiones en la escuela. Universidad Pedagógica Nacional.

Otálora, L. (2018). Estrategias de enseñanza para la Cátedra de la Paz en estudiantes de secundaria a partir de las Competencias Ciudadanas. Ciencia y Poder Aéreo, 13(1), 124-140. https://doi.org/10.18667/ cienciaypoderaereo. 592

Prada, M. (2020). Las competencias ciudadanas en la clase de Educación Física: entre las miradas desde la docencia y el discurso planteado en el currículo dominicano. Revista Educación, 45, (1). https://doi. org/10.15517/revedu.v45i1.41402

Peñalva, A., \& López-Goñi, J. (2014). Competencias ciudadanas en alumnado de magisterio: la competencia intercultural personal. Revista Electrónica Interuniversitaria de Formación del Profesorado, 17(2), 139153. https://doi.org/10.6018/reifop.17.2.196871

Pulido, Ó., \& Acuña, O. (2014). De la universidad de las profesiones a la universidad para el desarrollo 1953 -1974. Praxis \& Saber 5(10), 219-243.

Quintero, M., \& Molano, M. (2009). Concepciones y creencias acerca de las competencias en Colombia: una investigación acción desde la teoría crítica de la educación. Revista Centro de Investigación, 8(32), 39-55. 
Quiroz, R., \& Jaramillo, O. (2009). Formación ciudadana y educación cívica: ¿cuestión de actualidad o resignificación? Revista de Teoría y Didáctica de las Ciencias Sociales, (14), 123-138.

Ramos, C., Nieto, A., \& Chaux, E. (2007). Aulas en paz: resultados preliminares de un programa multicomponente. Revista Interamericana de Educación para la Democracia, 1(1), 35-56.

Rendón, M. (2012). Las convivencias y las competencias ciudadanas en la universidad. Uni-p/uri/versidad, 12(2), 57-72. https://revistas.udea.edu.co/index.php/unip/article/view/14436

República de Colombia. (1991). Constitución Política de la República de Colombia. http://www. secretariasenado.gov.co/index.php/constitucion-politica

Restrepo, J. (2006). Estándares básicos en competencias ciudadanas: una aproximación al problema de la formación ciudadana en Colombia. Papel Político, 17(1), 137-175.

Rincón, D. (2017). Formación ciudadana en el currículo escolar en Colombia. Desde la pedagogía católica hasta las competencias ciudadanas [Tesis de pregrado, Universidad Santo Tomás]. Repositorio Institucional Universidad Santo Tomás. http://hdl.handle.net/11634/10223

Rodríguez, A., Ruiz, S., \& Guerra, Y. (2007). Competencias ciudadanas aplicadas a la educación en Colombia. Revista Educación y Desarrollo Social, 1(1), 140-157.

Rodríguez, G. (2013). Pactos de aula para el desarrollo de competencias ciudadanas [Tesis de maestría, Universidad Tecnológica de Pereira]. Repositorio Institucional Universidad Tecnológica de Pereira. http://hdl.handle.net/11059/3118

Rodríguez, H. (2007). El paradigma de las competencias hacia la educación superior. Revista Facultad de Ciencias Económicas: Investigación y Reflexión, 15(1), 145-165

Rojas, J., Jiménez-Delgado, A., Jiménez-Jiménez, B., López-Delgado, I., Ospino-Ortiz, N., Cabarcas-Masson, M., Paba-Ruíz, M., \& Navarro-Ramírez, L. (2018). Investigación como estrategia pedagógica para la convivencia escolar a través de competencias ciudadanas. Cultura Educación y Sociedad, 9(3), 291302. https://doi.org/10.17981/cultedusoc.9.3.2018.33

Rojas, R. (2018). Formación en competencias ciudadanas: un referente para el debido proceso de una cultura de paz y el desarrollo humano integral en las Instituciones Educativas de Básica Primaria [Trabajo de grado de especialización, Universidad Nacional Abierta y a Distancia]. Repositorio Institucional UNAD. https://repository.unad.edu.co/handle/10596/28032

Ruiz, A., \& Chaux, E. (2005). La formación de competencias ciudadanas ( $1^{\mathrm{a}}$ ed.). Asociación Colombiana de Facultades de Educación.

Salas, K., \& Cómbita, H. (2017). Análisis de la convivencia escolar desde la perspectiva psicológica, legal y pedagógica en Colombia. Cultura, Educación y Sociedad, 8(2), 79-92. https://doi.org/10.17981/ cultedusoc.8.2.2017.06

Salmerón, C. (2010). Desarrollo de la competencia social y ciudadana a través del aprendizaje cooperativo [Tesis doctoral, Universidad de Granada]. https://digibug.ugr.es/handle/10481/5667?show=full

Santander, W., Ovalle, V., Cervantes, M., Villamil, J., \& Rivera, A. (2018). Las competencias ciudadanas como generadoras de cultura ambiental. Cultura Educación y Sociedad, 9(2), 67-76.

Tiana, A. (2000). El proyecto MANES y la investigación histórica sobre los manuales escolares (siglos XIX y XX). Historia de la Educación, 19, 179-194.

Tiana, A. (2005). La investigación histórica sobre los manuales escolares en España: el Proyecto MANES. Clío \& Asociados. La Historia Enseñada, 1(4), 101-119.

Torney-Purta, J., Lehmann, R., Oswald, H., \& Schulz, W. (Eds.). (1999). La educación cívica y ciudadanía Proyecto de educación cívica de la IEA. Asociación Internacional para la Evaluación del Logro Educativo

Torres, C., \& Reyes, E. (2015). Subjetivación en la escuela: una mirada crítica al programa de competencias ciudadanas. Educación y Ciencia, (18), 11-28.

Vega, M., \& Castro, V. (2006). Cuentos ciudadanos, desarrollo de competencias ciudadanas y reconstrucción de imaginarios de ciudad y ciudadanía, a través del periodismo escolar. Investigación y Desarrollo, 14(2), 330-351.

Villera, E., \& Lobo, J. (2017). Las competencias ciudadanas en el ambiente escolar de la institución educativa de Buenos Aires de Montería, Córdoba [Tesis de maestría, Universidad Pedagógica Nacional]. Repositorio Institucional UPN. https://hdl.handle.net/20.500.12209/719 